\title{
Low seroprevalence of West Nile virus in blood donors from Catalonia (Spain)
}

M. Piron ${ }^{1}$, A. Plasencia ${ }^{2}$, E. Fleta-Soriano ${ }^{3}$, A. Martinez ${ }^{4}$, J.P. Martinez ${ }^{3}$, N. Torner ${ }^{4}$, S. Sauleda $^{1}$, A.Meyerhans ${ }^{3,5}$, J. Escalé ${ }^{6}$, A. Trilla ${ }^{7}$, T. Pumarola ${ }^{8}$, and MJ. Martinez ${ }^{2,6 *}$

1 Transfusion safety laboratory, Catalonian Blood and Tissue

Bank (BST), Barcelona, Spain

2 ISGlobal, Barcelona Ctr. Int. Health Res. (CRESIB), Hospital Clínic -Universitat de Barcelona, Barcelona, Spain

3 Infection Biology Group, Department of Experimental and Health

Sciences, Universitat Pompeu Fabra, Barcelona, Spain

4 Public Health Agency of Catalonia; Generalitat of Catalonia, Barcelona, Spain

5 Institució Catalana de Recerca i Estudis Avançats (ICREA), Barcelona, Spain

6 Department of Clinical Microbiology, Hospital Clinic, Barcelona, Spain

7 Department of Preventive Medicine, Hospital Clinic, Barcelona, Spain

8 Microbiology Department, Hospital Universitario Vall d’Hebron, Barcelona, Spain

Keywords: West Nile virus, seroprevalence, Northeastern Spain

Running tittle: West Nile seroprevalence, Northeastern Spain

*Corresponding author: Miguel J. Martínez. Department of Clinical Microbiology, ISGlobal, Barcelona Ctr. Int. Health Res. (CRESIB), Hospital Clínic Universitat de Barcelona, Barcelona, Spain, Villarroel 170, 08036-Barcelona, Spain. Tel. +34 93 2275522; Fax: +34 93 2279372. E-mail address: myoldi@clinic.ub.es 


\section{Introduction}

West Nile Virus (WNV) is an emerging flavivirus maintained in nature in an enzootic cycle between mosquitoes of the genus Culex and birds. Humans are infected mainly by the bites of infected mosquitoes, although other routes of transmission such as blood transfusion and organ transplantation have been reported (Iwamoto et al. 2003). WNV is commonly found in Africa,Europe, the Middle East, North America and West Asia. Serological studies suggest that WNV may have circulated in Europe earlier than the first documented outbreak inFrance in 1962. Since then, a number of human outbreaks have been described in European countries over the last two decades (Zeller et al. 2004). Greece and Italy have reported human WNV infections for the last three and five consecutive years respectively (ECDC 2013). In Spain, early studies using hemaglutinin inhibition techniques suggested that WNV or closely related flaviviruses circulated in some parts of the country during the 1970's (Gonzalez et al. 1977; Lozano et al. 1998). Clear evidence of WNV activity in Spain has been accumulating since the year 2000, including detection of neutralizing antibodies in birds, horses, detection of WNV RNA and virus isolation in eagles (Jimenez-Clavero et al. 2010), and the description of a single human case of WNV neurologic disease in 2004 (Kaptoul et al. 2007). In 2010, the first outbreak of WNV was reported in Southern Spain, affecting more than 50 horses and 2 humans with WNV neurologic disease (Garcia-Bocanegra et al. 2011). In Catalonia, (Northeastern Spain) three main wetlands that serve as breeding sites for wild birds as well as mosquito vectors are present (Ebro river delta, Lobregat river delta and the and the Empordà wetlands). The region is considered a high-risk area for WNV and other arboviruses transmission. We conducted a WNV seroprevalence study in Catalonia to investigate if WNV had been circulating to a significant extent and to provide baseline seroprevalence data for this region for future studies 


\section{Materials and Methods}

Between November 2010 and February 2011 we collected samples from blood donors in 14 municipalities of the 4 catalonian provinces, selected according to the proximity of the wetlands and to Culex mosquitoes presence (Table 1). Basic demographic information of the donors was collected before anonymization of the samples with study numbers. The samples were screened by a commercial Enzymelinked Immunosorbent Assay (ELISA) (Focus Diagnostics) for the presence of IgG antibodies against WNV. A microneutralization protocol using two-fold sample dilutions (1:10-1:640) and 100 TCID50 of WNV strain NY99 in Vero cells was used as a confirmatory assay. As only WNV lineage 1 has been detected in humans, horses and birds in Spain, this lineage was selected for the neutralization protocol. Serum samples from 800 blood donors were collected and stored frozen until testing.

\section{Results and Discussion}

The median age of the blood donors was 45 years, ranging from 18 to 69 years; $45 \%$ were female. Most donors (97\%) were born in Spain. Fifty-two samples were positive and twelve showed equivocal results by ELISA. Among these samples, only one resulted positive in the microneutralization assay (titer 1/40), and was negative for IgM antibodies by capture ELISA (Diagnostics). This sole positive sample was obtained from a blood donor native of Pakistan, a country where WNV and other flaviviruses such as Japanese encephalitis or dengue circulate (Hayes et al. 1982, Igarashi et al. 1994, Rafique et al. 2015). Given the low neutralization titer and the country of origin of the donor, the serological results suggest past infection by WNV or a closely related flavivirus acquired not necessarily in our region. Cross reactivity with other flaviviruses infection or vaccination (Hogrefe et al. 2004) and even with other unrelated viruses (Focus Diagnostics 2011) has been reported to occur with WNV ELISA's and therefore seroneutralization is needed to be performed as a confirmatory assay. More than $75 \%$ of the samples analyzed by neutralization showed 
low (<2) ELISA values (cut off: 1.1), whereas the sample with a neutralization titer of 1/40 exhibited the highest ELISA value (5.7) of all samples.

Similar WNV seroprevalence studies in blood donors have been performed in other European countries such as France (Charrel et al. 2001) or Italy (Pezzotti et al. 2011, Pierro et al. 2013), detecting 0.76 and $0.78 \%$ samples with neutralizing antibodies respectively, although these studies were conducted in areas with previous documented circulation of the virus. The last WNV seroprevalence study in Catalonia was performed in 2001, focussing on one of the three wetlands in the region (Ebro river delta). From 992 participants, 2 samples were positive by seroneutralization (Bofill et al. 2006). An ecological surveillance for WNV in Catalonia has been recently described (Alba et al. 2014). Horses, sentinel chickens, wild birds and mosquitoes were analyzed from 2007 to 2011. No evidence of WNV infection was found in domestic animals or mosquitoes. However, positive serological tests were obtained in wild birds during 2010 and2011, suggesting that WNV or a closely related flavivirus may have circulated in the region. Our seroprevalence results correlate with the absence of acute WNV infections in the animal surveillance and the absence of reported human cases in Catalonia. Moreover, no evidence of WNV infection was detected in 50 human cases of meningoencephalitis of unknown aetiology tested at our laboratory during the last three years(unpublished data). Taken together, it seems that transmission of WNV and human infections in Catalonia are rare. However, WNV activity has remained in Southern Spain since the 2010 outbreak and the factors driving the emergence of WNV are not completely understood. Therefore, surveillance and preparedness in our region should be continued and strengthened. Our seroprevalence study may be useful as a baseline reference data for future monitoring of WNV presence in the Catalonia region.

\section{Author Disclosure Statement}

No competing financial interests exist. 


\section{References}

Alba, A., A. Allepuz, et al. (2014). "Ecological Surveillance for West Nile in Catalonia (Spain), Learning from a Five-Year Period of Follow-up." Zoonoses Public Health. May;61(3):181-91

Bofill, D., C. Domingo, et al. (2006). "Human West Nile virus infection, Catalonia, Spain." Emerg Infect Dis 12(7): 1163-4.

Charrel RN, Lamballerie X de, et al. (2001). "Prevalence of antibody against West Nile virus in volunteer blood donors living in southeastern France.” Transfusion; 41(10):1320-1321.

European Centre for Disease Prevention and Control. West Nile virus risk assessment tool Stockholm:

ECDC; (2013). (Accesible at http://ecdc.europa.eu/en/publications/publications/westnile-virus-risk-assessment-tool.pdf)

Focus Diagnostics. (2011). "West Nile Virus IgG DxSelect ${ }^{\mathrm{TM}}$. Product Insert. Focus Diagnostics." (Accesible at https://www.focusdx.com/product/EL0300G/ous)

Garcia-Bocanegra, I., J. A. Jaen-Tellez, et al. (2011). "West Nile fever outbreak in horses and humans, Spain, 2010." Emerg Infect Dis 17(12): 2397-9.

Gonzalez, M. T. and A. R. Filipe (1977). "Antibodies to arboviruses in northwestern Spain." Am J Trop Med Hyg 26(4): 792-7.

Hayes CG, Baqar S, et al. (1982). "West Nile virus in Pakistan. 1. Sero-epidemiological studies in Punjab Province.” Trans R Soc Trop Med Hyg. 1982; 76(4):431-6. 
Hogrefe, W. R., R. Moore, et al. (2004). "Performance of immunoglobulin G (IgG) and IgM enzyme-linked immunosorbent assays using a West Nile virus recombinant antigen (preM/E) for detection of West Nile virus- and other flavivirusspecific antibodies." J Clin Microbiol42(10): 4641-8.

Igarashi A, Tanaka M, et al. (1994) “Detection of west Nile and Japanese encephalitis viral genome sequences in cerebrospinal fluid from acute encephalitis cases in Karachi, Pakistan.” Microbiol Immunol.; 38(10):827-30.

Iwamoto, M., D. B. Jernigan, et al. (2003). "Transmission of West Nile virus from an organ donor to four transplant recipients." N Engl J Med 348(22): 2196-203.

Jimenez-Clavero, M. A., F. Llorente, et al. (2010). "West Nile virus serosurveillance in horses in Donana, Spain, 2005 to 2008." Vet Rec 167(10): 379-80.

Kaptoul D, Viladrich PF, et al. (2007). "West Nile virus in Spain: report of the first diagnosed case (in Spain) in a human with aseptic meningitis.” Scand J Infect Dis.;39(1):70-1.

Lozano, A. and A. R. Filipe (1998). "[Antibodies against the West Nile virus and other arthropod-transmitted viruses in the Ebro Delta region]." Rev Esp Salud Publica 72(3): 245-50.

Pezzotti P, Piovesan C, et al. (2011). "Prevalence of IgM and IgG antibodies to West Nile virus among blood donors in an affected area of north-eastern Italy, summer 2009” . Euro Surveill. ;16(10) 
Pierro A, Gaibani P, et al. (2013). “Detection of specific antibodies against West Nile and Usutu viruses inhealthy blood donors in northern Italy, 20102011.” Clin Microbiol Infect ; 19(10):E451-3.

Rafique I, Nadeem Saqib MA, et al. (2015). “Economic burden of dengue in four major cities of Pakistan during 2011”. J Pak Med Assoc; 65(3):256-9.

Zeller, H. G. and I. Schuffenecker (2004). "West Nile virus: an overview of its spread in Europe and the Mediterranean basin in contrast to its spread in the Americas." Eur J Clin Microbiol Infect Dis 23(3): 147-56.

Table1. Distribution of samples tested by location

\begin{tabular}{|l|l|c|}
\hline Province (wetland) & \multicolumn{1}{|c|}{ Municipality } & No ofsamples \\
\hline Barcelona & El Prat de Llobregat & 70 \\
(Llobregat river delta) $)$ & Viladecans & 70 \\
& Gavà & 50 \\
& Sant Boi & 90 \\
\hline de Llobregat & 39 \\
\hline Girona & Sils & 15 \\
(Empordá wetlands) & Castelló d'Empúries & 20 \\
& L'Escala & 50 \\
\hline Tarragona & Roses & 98 \\
(Ebro river delta) & Deltebre & 24 \\
& L'Ampolla & 44 \\
& Sant & 33 \\
& Jaume d'Enveja & 63 \\
\hline Lleida (none) & Camarles & 134 \\
\hline \multicolumn{2}{|c|}{ L'Aldea } & $\mathbf{8 0 0}$
\end{tabular}

